\title{
PRESTARLIKE FUNCTIONS WITH NEGATIVE COEFFICIENTS
}

\author{
H. SILVERMAN* \\ Department of Mathematics \\ College of Charleston \\ Charleston, South Carolina 29401 \\ E. M. SILVIA \\ Department of Mathematics \\ University of California, Davis \\ Davis, California 95616 \\ (Received December 4, 1978)
}

ABSTRACT. The extreme points for prestarlike functions having negative coefficients are determined. Coefficient, distortion and radii of univalence, starlikeness, and convexity theorems are also obtained.

KEY WORDS AND PHRASES. Prestarlike of order $\alpha$, starlike of order $\alpha$, radius of univalence, radius of convexity, extreme points.

AMS (MOS) SUBJECT CLASSIFICATION (1970) CODES. Primary 30A32, Primary 30A34, Secondary 30440 .

1. INTRODUCTION.

Let $S$ denote the class of functions normalized by $f(0)=f^{\prime}(0)-1=0$ that are analytic and univalent in the unit disk $U$. Given $\alpha, 0 \leq \alpha \leq 1$, a function $\mathrm{f} \in \mathrm{S}$ is said to be in the class of functions starlike of order $\alpha$, denoted by $\mathrm{S}^{*}(\alpha)$, 
if

$$
\operatorname{Re}\left\{\mathrm{zf}^{\prime}(z) / f(z)\right\} \geq \alpha \quad(z \in U),
$$

and is said to be in the class of functions convex of order $\alpha$, denoted by $K(\alpha)$, if

$$
\operatorname{Re}\left\{1+z f^{\prime \prime}(z) / f^{\prime}(z)\right\} \geq \alpha \quad(z \in U) .
$$

Further, let $T$ and $T *[\alpha]$ denote the subclasses of $S$ and $S *(\alpha)$, respectively, whose elements can be expressed in the form $f(z)=z-\Sigma_{n=2}^{\infty}\left|a_{n}\right| z^{n}$.

The convolution or Hadamard product of two power series $f(z)=\Sigma_{n=0}^{\infty} a_{n} z^{n}$ and $g(z)=\Sigma_{n=0}^{\infty} b_{n} z^{n}$ is defined as the power series $(f * g)(z)=\Sigma_{n=0}^{\infty} a_{n} b_{n} z^{n}$. An analytic function normalized by $f(0)=f^{\prime}(0)-1=0$ is said to be in the class of functions prestarlike of order $\alpha, 0 \leq \alpha \leq 1$, denoted by $R_{\alpha}$, if $f * s_{\alpha} \in S *(\alpha)$ where $s_{\alpha}(z)=$ $z /(1-z)^{2(1-\alpha)}$. The function $s_{\alpha}$ is the well-known extremal function for the class $S *(\alpha)$. In the sequel, we let

$$
c(\alpha, n)=\frac{\pi_{k=2}^{n}(k-2 \alpha)}{(n-1) !} \quad(n=2,3, \ldots),
$$

so that $s_{\alpha}$ can be written in the form

$$
s_{\alpha}(z)=z+\Sigma_{n=2}^{\infty} C(\alpha, n) z^{n}
$$

Note that $C(\alpha, n)$ is a decreasing function of $\alpha$ with

$$
\lim _{n \rightarrow \infty} \mathrm{C}(\alpha, \mathrm{n})=\left\{\begin{array}{ll}
\infty, & \alpha<1 / 2 \\
1, & \alpha=1 / 2 \\
0, & \alpha>1 / 2
\end{array}\right\}
$$

The class $R_{\alpha}$ was introduced by Ruscheweyh [3], who showed that a necessary and sufficient condition for $f$ to be in $R_{\alpha}$ is that the functional 


$$
G(\alpha, z)=\frac{f(z) * \frac{s_{\alpha}(z)}{1-z}}{f(z) * s_{\alpha}(z)}
$$

satisfy

$$
\operatorname{Re} G(\alpha, z)>1 / 2 \quad(z \in U) .
$$

Since $s_{1}(z)=z$, we say that $f$ is prestarlike of order 1 if and only if $\operatorname{Re}\{f(z) / z\}>1 / 2(z \in U)$. Note that $R_{0}=K(0)$ and $R_{1 / 2}=S *(1 / 2)$. In [3] it was shown that

$$
R_{\alpha} \subset R_{B} \quad \text { for } 0 \leq \alpha<\beta \leq 1 \text {, }
$$

which generalizes the well-known result that $K(0) \subset S *(1 / 2)$.

In Section 2, we obtain a sufficient condition in terms of the modulus of the coefficients for a function to be in $R_{\alpha}$ and show that this condition is also necessary for the subclass

$$
R[\alpha]=R_{\alpha} \cap T \text {. }
$$

In Section 3, we obtain the extreme points for the closed convex hull of $R[\alpha]$ and use them to prove distortion and covering theorems. In Section 4, we determine the radii of univalence, starlikeness, and convexity for $R[\alpha]$. Finally, we find the smallest $\beta=\beta(\alpha)$ for which $T *[\alpha] \subset R[\beta], 0 \leq \alpha<1$.

2. COEFFICIENT INEQUALITIES FOR THE CLASS $R[\alpha]$.

We first obtain a relationship between the order of prestarlikeness of a function and the modulus of its coefficients.

THEOREM 1. Let $f(z)=z+\sum_{n=2}^{\infty} a_{n} z^{n}$. If

$$
\sum_{n=2}^{\infty} \frac{(n-\alpha) C(\alpha, n)}{1-\alpha}\left|a_{n}\right| \leq 1, \quad 0 \leq \alpha \leq 1,
$$

then $\mathrm{f} \in \mathrm{R}_{\alpha}$. 
REMARK. For the case $\alpha=1$ in the theorem and all subsequent results, the expression $(n-\alpha) C(\alpha, n) /(1-\alpha)$ is taken to be 2 , its limit as $\alpha \rightarrow 1^{-}$.

PROOF. An equivalent formulation of (1.3) is $|1 / G(\alpha, z)-1|<1,0 \leq \alpha \leq 1$. For $s_{\alpha}(z)=z+\sum_{n=2}^{\infty} C(\alpha, n) z^{n}$, we have $s_{\alpha}(z) /(1-z)=z+\sum_{n=2}^{\infty} \sigma(\alpha, n) z^{n}$ where $\sigma(\alpha, n)=1+\Sigma_{k=2}^{n} c(\alpha, k)$. Thus

$$
\begin{aligned}
\left|\frac{1}{G(\alpha, z)}-1\right| & =\left|\frac{\sum_{n=2}^{\infty} \sigma(\alpha, n-1) a_{n} z^{n}}{z+\sum_{n=2}^{\infty} \sigma(\alpha, n) a_{n} z^{n}}\right| \\
& \leq \frac{\sum_{n=2}^{\infty} \sigma(\alpha, n-1)\left|a_{n}\right|}{1-\sum_{n=2}^{\infty} \sigma(\alpha, n)\left|a_{n}\right|},
\end{aligned}
$$

which is bounded by 1 whenever (2.1) is satisfied.

The converse of Theorem 1 is also true for the class $R[\alpha]$, defined by (1.4). Since a necessary and sufficient condition [4] for

$f(z)=z-\sum_{n=2}^{\infty}\left|b_{n}\right| z^{n}$ to be in $T *[\alpha]$ is that

$$
\sum_{n=2}^{\infty}(n-\alpha)\left|b_{n}\right| \leq 1-\alpha
$$

we obtain

THEOREM 2. A function $f(z)=z-\sum_{n=2}^{\infty}\left|a_{n}\right| z^{n}$ is in $R[\alpha]$ if and only if

$$
\sum_{n=2}^{\infty} \frac{(n-\alpha) C(\alpha, n)}{1-\alpha}\left|a_{n}\right| \leq 1, \quad 0 \leq \alpha \leq 1
$$

COROLLARY. If $\mathrm{f}(\mathrm{z})=\mathrm{z}-\sum_{\mathrm{n}=2}^{\infty}\left|\mathrm{a}_{\mathrm{n}}\right| \mathrm{z}^{\mathrm{n}} \in \mathrm{R}[\alpha], 0 \leq \alpha \leq 1$, then $\left|a_{n}\right| \leq(1-\alpha) /(n-\alpha) c(\alpha, n)$, with equality only for functions of the form $z-(1-\alpha) z^{n} /(n-\alpha) C(\alpha, n)$.

We now determine the extreme points of this class. 


\section{EXTREME POINTS OF $\mathrm{R}[\alpha]$.}

For any compact family of analytic functions $\mathfrak{F}$, it is well known that the real part of any continuous linear functional over $\mathcal{F}$ is maximized (minimized) at one of the extreme points of the closed convex hull of $\mathcal{F}$. The solutions to several extremal problems in $\mathrm{R}[\alpha]$ follow easily from the extreme points for this class. In view of Theorem 2, we see that $R[\alpha]$ is a closed convex family. Thus, the extreme points are obtained in

THEOREM 3. Set $f_{1}(z)=z$ and $f_{n}(z)=z-(1-\alpha) z^{n} /(n-\alpha) c(\alpha, n), n=2,3, \ldots$. Then $f \in R[\alpha], 0 \leq \alpha \leq 1$, if and only if it can be expressed in the form $f(z)=\Sigma_{n=1}^{\infty} \lambda_{n} f_{n}(z)$ where $\lambda_{n} \geq 0$ and $\Sigma_{n=1}^{\infty} \lambda_{n}=1$.

PROOF. Suppose $f(z)=\Sigma_{n=1}^{\infty} \lambda_{n} f_{n}(z)$. Then

$$
\sum_{n=2}^{\infty} \frac{(n-\alpha) C(\alpha, n)}{1-\alpha} \cdot \frac{\lambda_{n}(1-\alpha)}{(n-\alpha) C(\alpha, n)}=\sum_{n=2}^{\infty} \lambda_{n}=1-\lambda_{1} \leq 1
$$

Therefore, $f \in R[\alpha]$ by Theorem 2 .

Conversely, suppose $f(z)=z-\sum_{n=2}^{\infty}\left|a_{n}\right| z^{n} \in R[\alpha], 0 \leq \alpha \leq 1$. Then

$\left|a_{n}\right| \leq(1-\alpha) /(n-\alpha) C(\alpha, n), n=2,3, \ldots$. Set $\lambda_{n}=(n-\alpha) c(\alpha, n)\left|a_{n}\right| /(1-\alpha)$ and

$\lambda_{1}=1-\Sigma_{n=2}^{\infty} \lambda_{n}$. From Theorem 2, it follows that $\lambda_{1} \geq 0$. Since

$f(z)=\Sigma_{n=1}^{\infty} \lambda_{n} f(z)$, the proof is complete.

As an immediate consequence of Theorem 3, we obtain distortion theorems for the class $\mathrm{R}[\alpha]$.

THEOREM 4. If $f(z)=z-\sum_{n=2}^{\infty}\left|a_{n}\right| z^{n} \in R[\alpha], 0 \leq \alpha \leq 1$, then

$$
r-\frac{1}{2(2-\alpha)} r^{2} \leq|f(z)| \leq r+\frac{1}{2(2-\alpha)} r^{2} \quad(|z|=r),
$$

with equality only for $f_{2}(z)=z-\frac{1}{4-2 \alpha} z^{2}, \quad z= \pm r$. 
PROOF. From Theorem 3, we have

$$
r-\max _{n} \frac{1-\alpha}{(n-\alpha) C(\alpha, n)} r^{n} \leq|f(z)| \leq r+\max _{n} \frac{1-\alpha}{(n-\alpha) C(\alpha, n)} r^{n} .
$$

It suffices to show that $A(\alpha, n)=(1-\alpha) /(n-\alpha) C(\alpha, n)$ is a decreasing function of $n$. From (1.1), it follows that

$$
C(\alpha, n+1)=\frac{n+1-2 \alpha}{n} \cdot C(\alpha, n) \text {. }
$$

Therefore, we have $A(\alpha, n+1) \leq A(\alpha, n), n=2,3, \ldots$, whenever $(n+1-\alpha)(n+1-2 \alpha) \geq n(n-\alpha)$ This is equivalent to $(1-\alpha)[1+2(n-\alpha)] \geq 0$, which proves the result.

COROLLARY. The disk $|z|<1$ is mapped onto a domain that contains the disk $|w|<(3-2 \alpha) /(4-2 \alpha)$ for any $f \in R[\alpha], 0 \leq \alpha \leq 1$. The result is sharp, with extremal function

$$
f_{2}(z)=z-\frac{1}{2(2-\alpha)} z^{2} \in R[\alpha]
$$

PROOF. Let $\mathrm{r} \rightarrow 1^{-}$in Theorem 4 .

As a second application of Theorem 3, we have

THEOREM 5. If $f \in R[\alpha], 0 \leq \alpha \leq 1$, then $1-M(\alpha, r) \leq\left|f^{\prime}(z)\right| \leq 1+M(\alpha, r)$ $(|z|=r)$, where

$$
M(\alpha, r)=\max _{n} \frac{(1-\alpha) n}{(n-\alpha) C(\alpha, n)} r^{n-1}
$$

COROLLARY. If $\mathrm{f} \in \mathrm{R}[\alpha]$ with either $\mathrm{r} \leq 2 / 3$ or $\alpha \leq 1 / 2$, then

$$
1-\frac{1}{2-\alpha} r \leq\left|f^{\prime}(z)\right| \leq 1+\frac{1}{2-\alpha} r \quad(|z|=r) .
$$

PROOF. It suffices to show that 


$$
g(\alpha, r, n)=\frac{(1-\alpha) n r^{n-1}}{(n-\alpha) C(\alpha, n)}
$$

is a decreasing function of $n$. In view of (3.1), the inequality $g(\alpha, r, n+1) \leq g(\alpha, r, n)$ is equivalent to

$$
h(\alpha, r, n)=(1-r) n^{2}+[2-3 \alpha-(1-\alpha) r] n+(1-\alpha)(1-2 \alpha)+a r \geq 0
$$

Since, for $n$ fixed, $h$ is a decreasing function of $r$, we have

$$
h(\alpha, r, n) \geq h(\alpha, 1, n)=(1-2 \alpha) n+(1-\alpha)(1-2 \alpha)+\alpha \geq 0
$$

for $\alpha \leq 1 / 2$. Since $h$ is also a decreasing function of $\alpha$, th follows for $r \leq 2 / 3$ that

$$
h(\alpha, r, n) \geq h(1, r, n)=(1-r) n^{2}-n+r \geq h(1,2 / 3, n) \geq h(1,2 / 3,2)=0 .
$$

REMARKS. 1. Since $h(1, r, 2)=2-3 r<0$ for $r>2 / 3$, we have $g(1, r, 2)<g(1, r, 3)$. Thus the corollary will not be true for all $\alpha$ when $r>2 / 3$.

2. We next show that the corollary will not be true for all $r$ when $\alpha>1 / 2$. For each $\alpha, 1 / 2<\alpha<1$, we must find an $r=r(\alpha)$ such that $M(\alpha, r)>(1 /(2-\alpha)) r$. It suffices to show for $n=n(\alpha)$ sufficiently large that $(1-\alpha) n /(n-\alpha) C(\alpha, n)>1 /(2-\alpha)$, which is equivalent to

$$
C(\alpha, n)<\frac{(1-\alpha)(2-\alpha) n}{n-\alpha}
$$

Since $C(\alpha, n) \rightarrow 0$ for $\alpha>1 / 2$ and the right hand side of (3.2) is bounded below by $(1-\alpha)(2-\alpha)>0$, the result follows.

\section{RADII OF UNIVALENCE, STARLIKENESS, AND CONVEXITY.}

The functions in $\mathrm{R}[\alpha]$ for $0 \leq \alpha \leq 1 / 2$ are starlike of a positive order. The bound is given in 
THEOREM 6. If $\mathrm{f} \in \mathrm{R}[\alpha], 0 \leq \alpha \leq 1 / 2$, then $\mathrm{f} \in \mathrm{T} *[2(1-\alpha) /(3-2 \alpha)]$. The result is sharp, with extremal function

$$
f_{2}(z)=z-\frac{1}{2(2-\alpha)} z^{2}
$$

PROOF. From (2.2), it suffices to show that

$$
\sum_{n=2}^{\infty} \frac{(n-\alpha) C(\alpha, n)}{1-\alpha}\left|a_{n}\right| \leq 1
$$

implies

$$
\sum_{n=2}^{\infty} \frac{n-\frac{2(1-\alpha)}{3-2 \alpha}}{1-\frac{2(1-\alpha)}{3-2 \alpha}}\left|a_{n}\right| \leq 1
$$

This will follow if

$$
(3-2 \alpha) n-2(1-\alpha) \leq \frac{n-\alpha}{1-\alpha} C(\alpha, n)
$$

or, equivalently, when

$$
g(\alpha, n)=\frac{[(3-2 \alpha) n-2(1-\alpha)](1-\alpha)}{(n-\alpha) C(\alpha, n)} \leq 1 .
$$

Since $g(\alpha, 2)=1$, it suffices to show that $g(\alpha, n)$ is a decreasing function of $n$. In view of (3.1), the inequality $g(\alpha, n+1) \leq g(\alpha, n), n=2,3, \ldots$, is equivalent to

$$
\frac{(3-2 \alpha) n^{2}+n}{(n+1-\alpha)(n+1-2 \alpha)} \leq \frac{(3-2 \alpha) n-2(1-\alpha)}{n-\alpha} \text {. }
$$

This holds if and only if, for each fixed $\alpha$, we have

$$
h(n)=\left(4 \alpha^{2}-8 \alpha+3\right) n^{2}+\left(6 \alpha^{2}-4 \alpha^{3}-1\right) n+4 \alpha^{3}-10 \alpha^{2}+8 \alpha-2 \geq 0
$$

Note that $h(1)=0$ and

$$
h(n+1)-h(n)=2\left(4 \alpha^{2}-8 \alpha+3\right) n+2\left(1-4 \alpha+5 \alpha^{2}-2 \alpha^{3}\right)=A(\alpha) n+B(\alpha) \text {. }
$$


Since $A(\alpha) \geq 0$ and $B(\alpha) \geq 0$ for $0 \leq \alpha \leq 1 / 2$, the result follows.

REMARK. For $\alpha=0$, Theorem 6 reduces to the known result [4] that $R[0]=K[0] \subset T *[2 / 3]$.

When $\alpha>1 / 2, R[\alpha] \not S$. We will show that $g_{n}(z)=z-2 z^{n} / n \in R[\alpha]-S$ for $\mathrm{n}=\mathrm{n}(\alpha)$ sufficiently large. If $\mathrm{n} \geq 4$, then $\mathrm{g}_{\mathrm{n}} \in \mathrm{R}[1]$. If $1 / 2<\alpha<1$, then $\mathrm{g}_{\mathrm{n}}{ }^{*} \mathrm{~s}_{\alpha}=z-2 \mathrm{C}(\alpha, \mathrm{n}) z^{\mathrm{n}} / \mathrm{n}$. Taking (1.2) into account, we have $2 \mathrm{C}(\alpha, \mathrm{n}) / \mathrm{n} \leq(1-\alpha) /(\mathrm{n}-\alpha)$ for $\mathrm{n}$ sufficiently large, so that $\mathrm{g}_{\mathrm{n}}{ }^{\mathrm{s}_{\mathrm{s}}} \in \mathrm{T}{ }_{\alpha}[\alpha]$.

Therefore, $g_{n} \in R[\alpha]$.

We next determine the largest disk in which $R[\alpha]$ is univalent.

THEOREM 7. The radius of univalence and starlikeness for $R[\alpha], 1 / 2<\alpha \leq 1$, is

$$
r(\alpha)=\min _{n}\left\{\frac{(n-\alpha) C(\alpha, n)}{n(1-\alpha)}\right\}^{1 /(n-1)} .
$$

PROOF. For $f(z)=z-\sum_{n=2}^{\infty}\left|a_{n}\right| z^{n}$ in $R[\alpha]$, the inequality $\left|z f^{\prime} / f-1\right| \leqslant 1$ is valid for $|z| \leq r$ whenever $\sum_{n=2}^{\infty} n\left|a_{n}\right| r^{n-1} \leq 1$. In view of Theorem 2 , this is true if

$$
r \leq\left[\frac{(n-\alpha) C(\alpha, n)}{n(1-\alpha)}\right]^{1 /(n-1)} .
$$

Hence, $f$ is starlike for $|z| \leq r(\alpha)$. On the other hand, for some $n$ we have

$$
f_{n}^{\prime}(z)=1-\frac{n(1-\alpha)}{(n-\alpha) C(\alpha, n)} z^{n-1}=0 \text { when } z=r(\alpha)
$$

Thus $\mathrm{f}$ is not univalent (or starlike) for $|z| \leq r, r>r(\alpha)$.

COROLLARY. The radius of univalence and starlikeness for $R[1]$ is $1 / \sqrt[3]{2} \approx .794$

PROOF. We must show that 


$$
r(1)=\min _{n}\left(\frac{2}{n}\right)^{1 /(n-1)}=\frac{1}{\sqrt[3]{2}} .
$$

This can be found by differentiating $g(x)=(2 / x)^{1 /(x-1)}$ and observing that $g$ is decreasing for $2 \leq \mathrm{x} \leq \mathrm{x}_{0}$ and increasing for $\mathrm{x}>\mathrm{x}_{0}$, where $\mathrm{x}_{0}$ satisfies $4<\mathrm{x}_{0}<5$. Since $g(4)<g(5)$, the result follows.

Thus, for all $\alpha$, $f$ in $R[\alpha]$ is univalent and starlike when $|z|<1 / \sqrt[3]{2}$.

REMARK. MacGregor showed [2] that the radius of univalence and starlikeness for $\mathrm{R}_{1}$ is $1 / \sqrt{2} \approx .707$.

We will now obtain the radius of convexity for $R[\alpha]$.

THEOREM 8. If $\mathrm{f} \in \mathrm{R}[\alpha], 0 \leq \alpha \leq 1$, then $\mathrm{f}$ is convex in the disk

$$
|z|<r(\alpha)=\inf _{n}\left\{\frac{(n-\alpha) C(\alpha, n)}{n^{2}(1-\alpha)}\right\}^{1 /(n-1)} .
$$

The result is sharp, with the extremal function of the form

$$
f_{n}(z)=z-\frac{1-\alpha}{(n-\alpha) C(\alpha, n)} z^{n}
$$

for some $n$.

PROOF. For $f(z)=z-\sum_{n=2}^{\infty}\left|a_{n}\right| z^{n} \in R[\alpha]$, it suffices to show that $\left|z f^{\prime \prime}(z) / f^{\prime}(z)\right| \leq 1$ for $|z| \leq r(\alpha)$. We have

$$
\left|\frac{z f^{\prime \prime}(z)}{f^{\prime}(z)}\right|=\left|\frac{-\Sigma_{n=2}^{\infty} n(n-1)\left|a_{n}\right| z^{n-1}}{1-\Sigma_{n=2}^{\infty} n\left|a_{n}\right| z^{n-1}}\right| \leq \frac{\sum_{n=2}^{\infty} n(n-1)\left|a_{n}\right||z|^{n-1}}{1-\Sigma_{n=2}^{\infty} n\left|a_{n}\right||z|^{n-1}}
$$

which is bounded by 1 whenever $\sum_{n=2}^{\infty} n^{2}\left|a_{n}\right||z|^{n-1} \leq 1$. From Theorem 2, this will hold whenever 


$$
\mathrm{n}^{2}|\mathrm{z}|^{\mathrm{n}-1} \leq \frac{(\mathrm{n}-\alpha) \mathrm{C}(\alpha, \mathrm{n})}{1-\alpha}, \quad \mathrm{n}=2,3, \ldots
$$

or

$$
|z| \leq\left\{\frac{(n-\alpha) c(\alpha, n)}{n^{2}(1-\alpha)}\right\}^{1 /(n-1)}
$$

The result follows upon setting $|z|=r(\alpha)$ in (4.1).

Using arguments similiar to those in the corollary to Theorem 7 , we have the following

COROLLARY. The radius of convexity for $R[1]$ is $\sqrt{2} / 3 \approx .471$.

REMARK. The radius of convexity r.c. for $R_{1}$ is known to satisfy $.395 \leq$ r.c. $\leq .40$. See $[1]$.

We conclude with the determination of the smallest $\beta=B(\alpha)$ for which $T *[\alpha] \subset R[\beta]$

THEOREM 9. If $f \in T *[\alpha]$ then $f \in R[(2-3 \alpha) / 2(1-\alpha)]$ for $0 \leq \alpha \leq 1 / 2$, and f $\in R[1 / 2]$ for $1 / 2<\alpha<1$. The result is sharp, with extremal function

$$
z-\frac{1-\alpha}{2-\alpha} z^{2} \quad \text { for } 0 \leq \alpha \leq 1 / 2
$$

and of the form

$$
z-\frac{1-\alpha}{n-\alpha} z^{n} \quad \text { for } 1 / 2<\alpha<1 \text {. }
$$

PROOF. From Theorem 2 it suffices to show, for $0 \leq \alpha \leq 1 / 2$, that $\sum_{n=2}^{\infty}(n-\alpha)\left|a_{n}\right| /(1-\alpha) \leq 1$ implies $\sum_{n=2}^{\infty}(n-\beta) C(\beta, n)\left|a_{n}\right| /(1-\beta) \leq 1$, where $\beta=\beta(\alpha)=(2-3 \alpha) /(2-2 \alpha)$. This will follow if we can show that

$$
g(\alpha, \beta, n)=\frac{1-\alpha}{n-\alpha} \frac{n-\beta}{1-\beta} \quad C(\beta, n) \leq 1
$$

Since $g(\alpha, \beta, 2)=1$, it is sufficient to show that $g(\alpha, \beta, n)$ is a decreasing function 
of $\mathrm{n}$. In view of $(3.1), \mathrm{g}(\alpha, \beta, \mathrm{n}+1) \leq \mathrm{g}(\alpha, \beta, \mathrm{n})$ whenever

$$
\frac{(n+1-\beta)(n+1-2 \beta)}{n(n+1-\alpha)} \leq \frac{n-\beta}{n-\alpha},
$$

which is equivalent to

$$
\mathrm{p}(\alpha, \mathrm{n})=\frac{1-2 \alpha}{2(1-\alpha)^{2}}\left(2(1-\alpha) \mathrm{n}^{2}+\left(\alpha^{2}+2 \alpha-2\right) \mathrm{n}-\alpha^{2}\right) \geq 0 .
$$

However, since

$$
\mathrm{p}(\alpha, \mathrm{n}+1)-\mathrm{p}(\alpha, \mathrm{n})=\frac{1-2 \alpha}{2(1-\alpha)^{2}}\left(4(1-\alpha) \mathrm{n}+\alpha^{2}\right)
$$

is nonnegative for $0 \leq \alpha \leq 1 / 2$, the first inclusion is proved.

The inclusion relation $\mathrm{T} *\left[\alpha_{1}\right] \subset \mathrm{T} *\left[\alpha_{2}\right]$ for $\alpha_{1} \geq \alpha_{2}$ shows that $\mathrm{T} *[\alpha] \subset \mathrm{R}[1 / 2]$ for $\alpha>1 / 2$. But for any $\alpha<1$ and $\gamma<1 / 2$, we will show that

$$
f(z)=z-\frac{1-\alpha}{n-\alpha} z^{n} \in T^{*}[\alpha]-R[\gamma]
$$

for $n=n(\alpha, \gamma)$ sufficiently large. If $f \in R[\gamma]$, then

$$
\mathrm{f}_{\gamma}=z-\frac{1-\alpha}{n-\alpha} \quad C(\gamma, n) z^{n} \in T *[\gamma]
$$

This is true if and only if

$$
\frac{1-\alpha}{n-\alpha} \quad c(\gamma, n) \leq \frac{1-\gamma}{n-\gamma}
$$

or, equivalently, if

$$
C(\gamma, n) \leq\left(\frac{n-\alpha}{n-\gamma}\right)\left(\frac{1-\gamma}{1-\alpha}\right)
$$

Since $C(\gamma, n) \rightarrow \infty$ for $\gamma<1 / 2$ and the right hand side of (4.2) is bounded, the inequality is not true for $n$ sufficiently large.

REMARK. $T *[1]=\{z\} \subset R[0]$. 


\section{REFERENCES}

1. Campbe11, D. M., A Survey of Properties of the Convex Combination of Univalent Functions, Rocky Mt. J. 5, no. 4 (1975) 475-492.

2. MacGregor, T. H., The Radius of Convexity for Starlike Functions of Order $1 / 2$, Proc. Amer. Math. Soc. 14 (1963) 71-76.

3. St. Ruscheweyh, Linear Operators Between Classes of Prestarlike Functions, Comm. Math. Helv. 52 (1977) 497-509.

4. Silverman, H., Univalent Functions with Negative Coefficients, Proc. Amer. Math. Soc. 51 (1975) 109-116.

* The research of the first author was supported in part by grants from the American Philosophical Society and Sigma XI, and was completed while he was visiting at the University of California, Davis. 


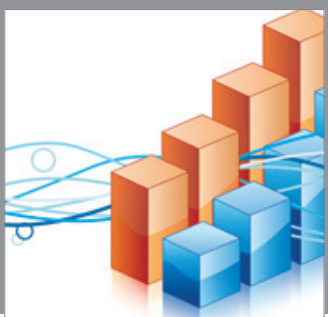

Advances in

Operations Research

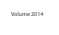

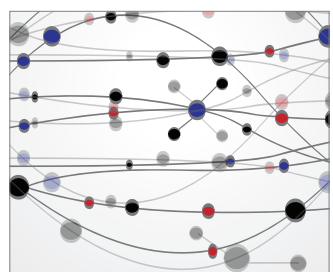

\section{The Scientific} World Journal
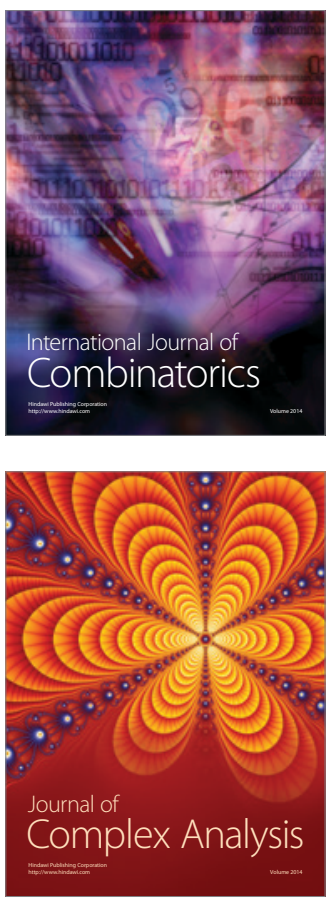

International Journal of

Mathematics and

Mathematical

Sciences
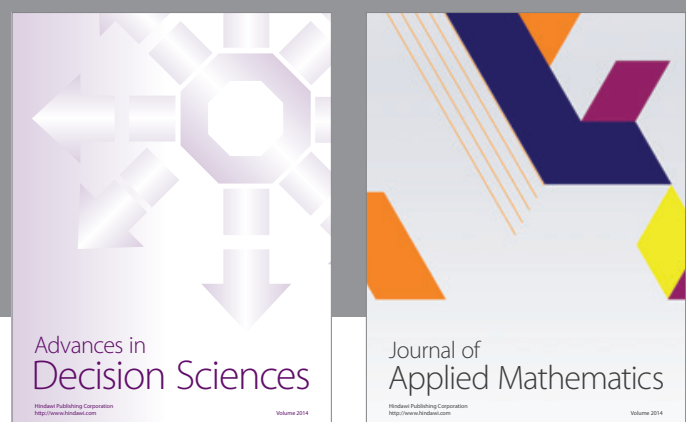

Journal of

Applied Mathematics
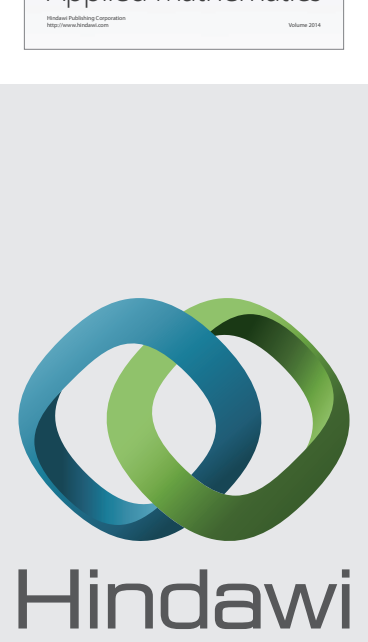

Submit your manuscripts at http://www.hindawi.com
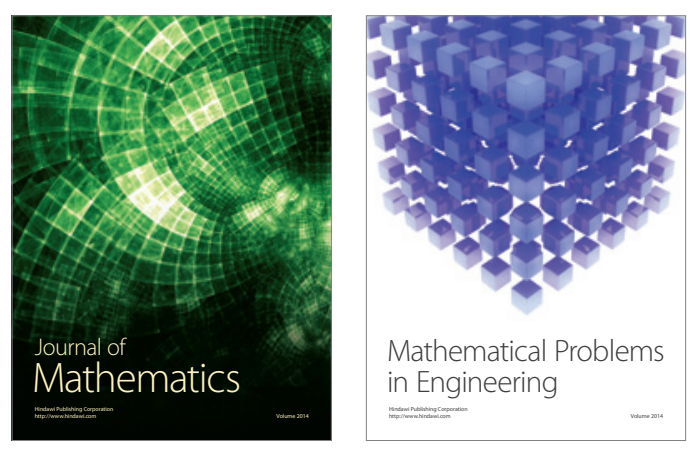

Mathematical Problems in Engineering
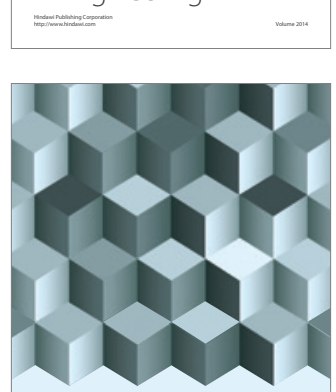

Journal of

Function Spaces
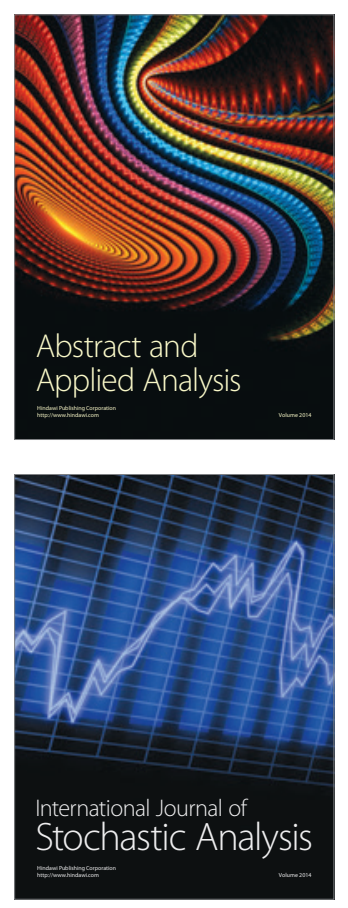

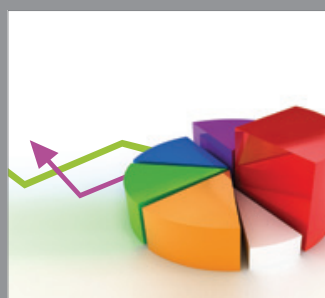

ournal of

Probability and Statistics

Promensencen
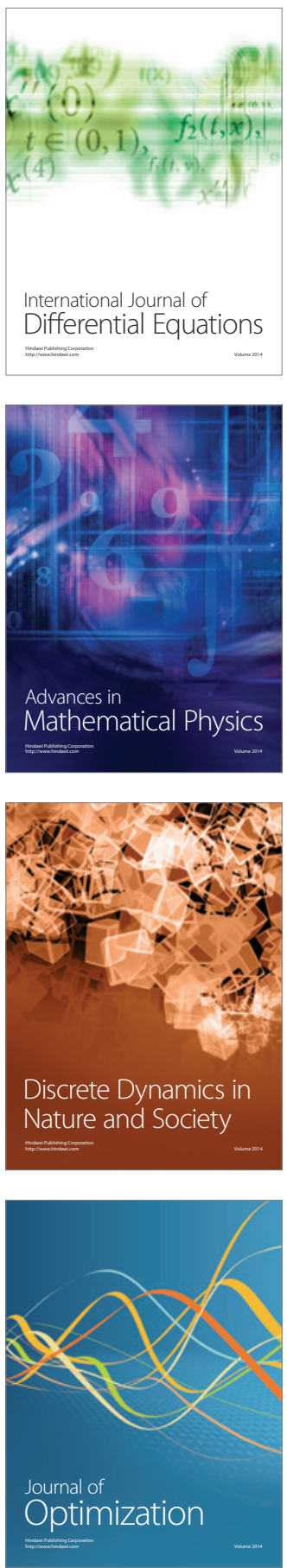